\title{
SUR LE THÉORÈME DE PRODUIT RELATIF AU DEGRÉ TOPOLOGIQUE D'UNE APPLICATION DANS L'ESPACE VECTORIEL TOPOLOGIQUE
}

\author{
MASUO HUKUHARA
}

Offert pour le soixantième anniversaire du Professeur Noshiro

$\S 1$. Soit $\boldsymbol{E}$ un espace vectoriel topologique localement convexe, $E$ un ensemble fermé dans $\boldsymbol{E}$ et $f$ une application complètement continue de $E$ dans $\boldsymbol{E}$. M. Nagumo a montré, ${ }^{1)}$ après avoir défini le degré topologique $d(b, E, I-f)$ de l'application $I-f$ de $E$ en un point $b \notin(I-f)(\partial E)$, la validité des propriétés importantes dont jouit le degré topologique. Mais dans sa démonstration du théorème de produit, il reste encore une lacune à combler.

En effet, soit $\Delta$ un ensemble ouvert contenant $(I-f)(E), \phi$ une application complètement continue de $\bar{\Delta}$ dans $\boldsymbol{E}$ et $c$ un point de $\boldsymbol{E}$ n'appartenant pas à

$$
(I-\phi)(\partial \Delta) \cup(I-\phi)(I-f)(\partial E) .
$$

Le théorème s'énonce alors comme il suit.

Si l'on désigne par $\Delta_{1}, \Delta_{2}, \ldots .$. les composants de l'ensemble ouvert $\Delta-(I-f)(\partial E)$, on $a$

$$
d(c, E,(I-\phi)(I-f))=\sum_{i} d\left(c, \bar{\Delta}_{i}, I-\phi\right) d\left(b_{i}, E, I-f\right),
$$

$b_{i}$ désignant un point quelconque de $\Delta_{i}$.

Désignons par $D_{k}$ l'ensemble des points $y \in \Delta$ tels que

$$
d(y, E, I-f)=k .
$$

Grâce à l'additivité du degré topologique, la relation (1) est équivalente à l'égalité

$$
d(c, E,(I-\phi)(I-f))=\sum_{k} k . d\left(c, \bar{D}_{k}, I-\phi\right) .
$$

$\partial D_{k}$ est une partie de l'ensemble

Received June 11, 1966.

1) Amer. J. Math., 73 (1951), 497-511. 
et l'image de celui-ci par $I-\phi$ ne contenant pas $c$, il existe un voisinage $\mathfrak{B}$ (convexe et symétrique de $o$ ) tel que

$$
\mathfrak{B}(c) \cap(I-\phi)\left(\partial D_{k}\right)=0
$$

Soit $\Gamma$ un compact $\supset \phi(\bar{\Delta})$. Il existe une application continue $S$ de $\Gamma$ dans un sous-espace à dimension finie telle que

$$
S y-y \in \mathfrak{B}
$$

pour $y \in \Gamma$. Soit $\mathfrak{B}$ l'ensemble des points $y \in \bar{\Delta}$ tels que

$$
(I-S \phi) y=c .
$$

$\mathfrak{B}$ est une partie compacte de $\Delta$ et on a

$$
\mathfrak{B} \cap(I-f)(\partial E)=0 .
$$

On peut en conclure l'existence d'un voisinage $U$ tel que

$$
U(\mathfrak{B}) \cap(I-f)(\partial E)=0 .
$$

Soit $C$ un compact $\supset f(E)$. Il existe une application continue $T$ de $C$ dans un sous-espace à dimension finie telle que

$$
T x-x \in U
$$

pour $x \in C$.

Posons

$$
f_{t}=(1-t) f+t T f .
$$

M. Nagumo suppose l'inclusion

$$
\left(I-f_{t}\right)(E) \subset \Delta,
$$

pour $t \in[0,1]$, ce qui n'est nulle part assuré. Si cette inclusion est assurée, sa démonstration est valide et son résultat est applicable. Cette remarque sera utile dans la suite.

§ 2. Nous voulons donc compléter sa démonstration. $B$ étant la plus grande partie compacte de $\Delta$ telle que $(I-\phi)(B)=c$, l'ensemble

$$
A=\{x \in E ;(I-f) x \in B\}
$$

est une partie compacte de l'intérieur $E^{0}$ de $E$. Prenons un voisinage $W$ tel 
que

$$
\Delta^{\prime}=(2 W)(B) \subset \Delta
$$

et puis un voisinage $V$ tel que

$$
\left.E^{\prime}=\overline{V(A}\right) \subset E^{0}, \quad(I-f)\left(E^{\prime}\right) \subset W(B) .
$$

D'après la définition de $E^{\prime}$, on a

$$
B \cap(I-f)\left(E-E^{\prime 0}\right)=0 .
$$

On peut donc trouver un voisinage $U$ contenu dans $W$ et tel que

$$
U(B) \cap(I-f)\left(E-E^{\prime 0}\right)=0 \text {. }
$$

Il est important de remarquer ici que rien n'empêche de remplacer $U$ par un voisinage plus petit. Prenons une application continue $T$ de $C$ dans un sous-espace à dimension finie telle que l'on ait (3). Défnissons ensuite la fonction $f_{t}$ par (4).

Si $x \in E$, on a

$$
\left(I-f_{t}\right) x-(I-f) x=\left(f-f_{t}\right) x=t(f-T f) x \in t U \subset U
$$

pour $t \in[0,1]$. Donc la relation

$$
\left(I-f_{t}\right) x \in B
$$

implique

$$
(I-f) x \in U(B)
$$

et la relation (5) implique $x \notin E-E^{\prime 0}$. On a par suite

$$
B \cap\left(I-f_{t}\right)\left(E-E^{\prime 0}\right)=0 \text {. }
$$

Si $x \in E^{\prime}$, on a

$$
\left(I-f_{t}\right) x \in U((I-f) x) \subset U(W(B)) \subset(2 W)(B),
$$

de sorte que l'on a

$$
\left(I-f_{t}\right)\left(E^{\prime}\right) \subset \Delta^{\prime}
$$

pour $t \in[0,1]$. Et puisqu'on peut supposer $U$ assez petit, la démonstration de M. Nagumo est valable si l'on restreint à $E^{\prime}$ le domaine de l'application $f$. Si donc on pose 


$$
D_{k}^{\prime}=\left\{y \in \Delta^{\prime} ; d\left(y, E^{\prime}, I-f\right)=k\right\},
$$

on a

$$
d\left(c, E^{\prime},(I-\phi)(I-f)\right)=\sum_{k} k \cdot d\left(c, \overline{D_{k}^{\prime}}, I-\phi\right)
$$

Or on a

$$
d(c, E,(I-\phi)(I-f))=d\left(c, E^{\prime},(I-\phi)(I-f)\right),
$$

car

$$
c \notin(I-\phi)\left(I-f_{t}\right)\left(E-E^{\prime 0}\right) .
$$

Par suite, pour que l'on puisse obtenir (2), il suffit de démontrer l'égalité

$$
d\left(c, \bar{D}_{k}, I-\phi\right)=d\left(c, \overline{D_{k}^{\prime}}, I-\phi\right) .
$$

Celle-ci est une conséquence immédiate de l'égalité

$$
B \cap D_{k}=B \cap D_{k}^{\prime} .
$$

Si $y \in B$, la relation (6) implique

$$
d\left(y, E, I-f_{t}\right)=d\left(y, E^{\prime}, I-f_{t}\right)
$$

pour $t \in[0,1]$. En posant $t=0$, on obtient

$$
d(y, E, I-f)=d\left(y, E^{\prime}, I-f\right),
$$

d'où découle l'égalité (7).

Institute of Mathematical Science

Kyoto University 